\title{
Review of: "Indirect impact of Covid-19 on hospital care pathways in Italy"
}

\author{
Antonino Salvatore Rubino
}

Potential competing interests: The author(s) declared that no potential competing interests exist.

The authors provided an excellent overview of the impact of COVID pandemic on primary care in Italy during lockdown and how this has increased the backlog at the end of the lockdown itself.

This unprecedented pandemic has wreaked havoc on the Italian healthcare system, who showed unprepared to face this burden of disease.

Institutions should pay attention to the limitation of our healthcare system, which need more empowerment to cope with the ordinary provision of care as well as the continuous impact of COVID on the daily practice 